\title{
Acute left ventricular insufficiency in a Burkitt Lymphoma patient with myocardial involvement and extensive local tumor cell lysis: a case report
}

\author{
Maren Schmiester ${ }^{{ }^{*}} \mathbb{D}$, Eva Tranter ${ }^{1}$, Alessandro Lorusso ${ }^{1}$, Florian Blaschke ${ }^{2}$, Dominik Geisel ${ }^{3}$, Lars Bullinger ${ }^{1}$, \\ Frederik Damm ${ }^{1}$ and II-Kang $\mathrm{Na}^{1}$
}

\begin{abstract}
Background: Burkitt lymphoma (BL) is a rare disease with the sporadic variant accounting for less than $1 \%$ of adult non-Hodgkin lymphomas. BL usually presents with an abdominal bulk, but extranodal disease affecting the bone marrow and central nervous system is common. Cardiac manifestations, however, are exceedingly rare, with less than 30 cases reported in the literature.

Case presentation: We report on a 54-year-old male patient with a six week-long history of paranasal sinus swelling, fatigue and dyspnea on exertion. Stage IV sporadic BL with extensive lymphonodal and cardiovascular involvement was diagnosed. Manifestations included supra- and infradiaphragmatic lymphadenopathy as well as infiltration of the aortic root, the pericardium, the right atrium and the right ventricle. EBV-reactivation was detected, which is uncommon in the sporadic subtype. After initial full-dose chemotherapy with very good BL control, the patient developed acute, but fully reversible cardiac insufficiency. Myocardial lymphoma involvement receded completely during the following two therapy cycles, while cardiac function periodically deteriorated shortly after chemotherapy administration and quickly recovered thereafter. Interestingly, the decline in cardiac function lessened with decreasing myocardial lymphoma manifestation. Once the cardiovascular BL infiltration was resolved, cardiac function remained stable throughout further treatment. Following seven cycles of chemotherapy and mediastinal radiation, the patient is now in continued complete remission.
\end{abstract}

Conclusions: Although rare, cardiac involvement in BL can quickly become life-threatening due to rapid lymphoma doubling time and should therefore be considered at initial diagnosis. This case suggests an association between myocardial infiltration, chemotherapy associated tumor cell lysis and transient deterioration of cardiac function until the damage caused by the underlying lymphoma could be restored. While additional studies are needed to further elucidate the mechanisms of acute cardiac insufficiency due to lymphoma lysis in the infiltrated structures, prompt BL control and full recovery of the patient supports courageous treatment start despite extensive cardiovascular involvement.

\footnotetext{
*Correspondence: maren.schmiester@charite.de

${ }^{1}$ Department of Hematology, Oncology, and Tumor Immunology,

Charité-Universitätsmedizin Berlin, Corporate Member of Freie Universität

Berlin and Humboldt-Universität zu Berlin, Berlin, Germany

Full list of author information is available at the end of the article
} 
Keywords: Burkitt Lymphoma, Case report, Cardiac neoplasms, Myocardial lymphoma involvement, Acute cardiac insufficiency

\section{Background}

Burkitt lymphomas (BL) are rare, highly aggressive hematologic neoplasms accounting for less than $1 \%$ of all nonHodgkin lymphomas (NHL) [1]. They are characterized by a marked proliferative activity as well as translocation and constitutive activation of the MYC oncogene. Three distinct clinical variants have been described, with the endemic form occurring in association with EpsteinBarr-Virus (EBV) infection in the equatorial regions of Africa, the immunodeficiency-associated form occurring as a manifestation of HIV infection and the sporadic form occurring non-endemically globally. While EBV-association is found in $95 \%$ of endemic cases, it is very uncommon in sporadic BL [2]. Payer's plaques of the ileocecal region are the most frequent site of origin in this subtype, resulting in an abdominal tumor [3]. Cardiac manifestations, on the other hand, are extremely rare [4]. Indeed, there are less than 30 reports published in the English literature as of now. In the few case studies available, more than a third of affected patients died of the disease within the first days after diagnosis [5], underscoring the need for swift diagnosis and treatment. Interestingly, the incidence of cardiac involvement across all NHL types appears much higher with reported ranges of $10-20 \%$. This affectation is often only discovered on autopsy, suggesting a lesser clinical relevance in these lymphomas as compared to BL [6-8].

Here, we present the case of a patient with BL who displayed extensive cardiovascular manifestations and a rapidly progressing disease with a life-threatening situation at the time of diagnosis. During chemotherapy, the patient experienced transient acute cardiac insufficiency, perhaps due to myocardial tumor cell lysis. Cardiac function fully recovered alongside with chemotherapy induced tumor control.

\section{Case presentation}

An otherwise healthy 54-year-old Caucasian male presented to our clinic with a six week-long history of paranasal sinus swelling, fatigue and dyspnea on exertion. Fever and weight loss were denied. Additional relevant findings on physical examination included tachycardia of 110/min and muffled heart sounds. On room air, oxygen saturation was normal (97\%) and respiratory rate was not elevated. No spleno- or hepatomegaly was palpable. Left-sided submandibular lymph node enlargement was present, other lymph nodes were not enlarged. Discrete congestion of cervical veins was seen, with the right side being more prominent. Slight bilateral ankle edema was noted. Neurological examination showed no abnormalities. Eastern Cooperative Oncology Group (ECOG) performance status was estimated at 2 [9].

Relevant laboratory findings included an elevated lactate dehydrogenase level (LDH) of $620 \mathrm{U} / 1$ (reference $<250 \mathrm{U} / \mathrm{l}$ ) and EBV copies of 395,000/ml. Testing for HIV was negative. A 12-lead electrocardiogram showed low QRS voltages and sinus tachycardia.

Computed tomography (CT) scans of the head and neck, chest, abdomen and pelvis showed cervical, mediastinal, paraaortic and retroperitoneal lymphadenopathy as well as an infiltration of the frontal, maxillary and sphenoidal sinuses by a suspected lymphoma. Notably, the mediastinal adenopathy showed encasement of the aortic root, the coronary arteries and an infiltration of the pericardium, the right ventricle and the right atrium (Fig. 1A-D).

An initial transthoracic echocardiography (TTE) revealed a hemodynamically relevant, circumferential pericardial effusion of about $2 \mathrm{~cm}$ with consecutive diastolic collapse of the right atrium. Left ventricular ejection fraction (LVEF) was normal at $60 \%$. The assessment of the right ventricular (RV) function was compromised by pericardial effusion; longitudinal function was normal (tricuspid lateral annular peak systolic velocity $0.23 \mathrm{~m} / \mathrm{s}$, tricuspid annular plane systolic excursion $23 \mathrm{~mm}$ ) and the proximal wall of the right ventricular outflow tract (RVOT) showed a thickening of $>1 \mathrm{~cm}$ with diminished local contractility. In addition, a solid right atrial mass of $13 \times 22 \mathrm{~mm}$ was seen, and possible differential diagnoses of intra-cardiac lymphoma, thrombus or myxoma were discussed (Fig. 1E).

Pericardiocentesis allowed the drainage of $1200 \mathrm{ml}$ of effusion over the course of three days. Cardiovascular magnetic resonance imaging was performed to analyze the extent of cardiac involvement in more detail. Here, pericardial and myocardial infiltration of the right atrium and ventricle and infiltration of the aortic root and was confirmed (Fig. 1F). The etiology of the right atrial mass remained unclear. As the risk of severe bleeding under chemotherapy and extensive vascular infiltration was assessed as relevant, only low-dose anticoagulation with low-molecular-weight heparin was initiated.

Biopsies were obtained from the paranasal sinuses, a submandibular lymph node and the bone marrow. A spinal tap was also performed, which showed no pathological findings. The patient was started on a prephase 

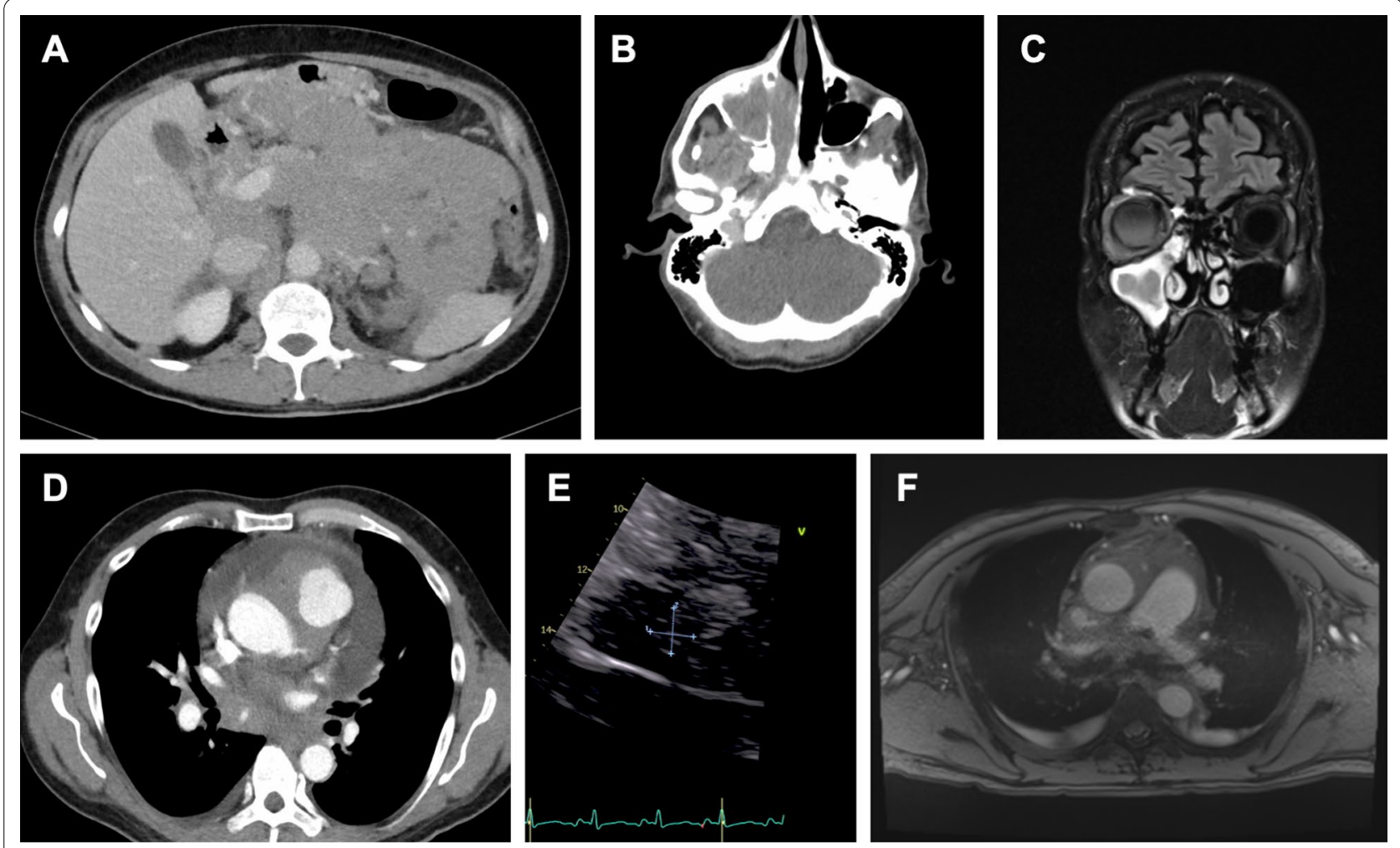

Fig. 1 CT and MR imaging reveal extensive BL manifestations. A Contrast-enhanced CT, large abdominal manifestation with infiltration of pancreas and mesenteric root. B and C Contrast-enhanced skull CT and MRI, destruction of posterior wall of right maxillary sinus by BL manifestation. D Contrast-enhanced chest CT, mediastinal manifestation with infiltration of supraaortic vessels. $\mathbf{E}$ TTE revealing a solid mass of $2.2 \times 1.3 \mathrm{~cm}$ of the right atrium. F Cardiac MRI: mediastinal and pericardial mass with infiltration of pericardium and bilateral pleural effusion

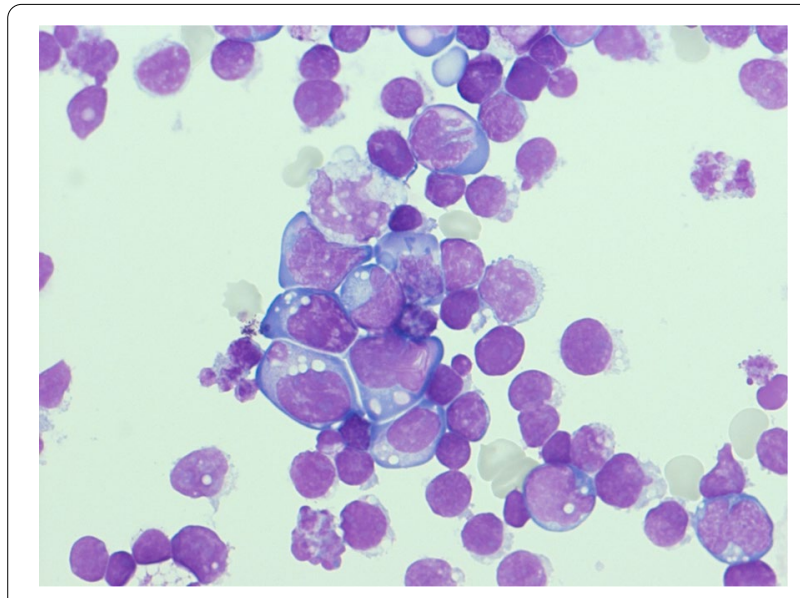

Fig. 2 Morphologic analysis of the pericardial effusion. Typical medium sized Burkitt lymphoma cells with cytoplasmic vacuoles

treatment with prednisone $(100 \mathrm{mg} /$ day for 5 days $)$ and cyclophosphamide ( $200 \mathrm{mg} / \mathrm{m}^{2}$ for 3 days).

Morphologic (Fig. 2) and flow cytometric analysis of the pericardial effusion described a mature B-cell neoplasia with an immunophenotype of $\mathrm{CD} 7 \mathrm{~b}^{+}$, $\mathrm{CD} 19^{+}, \mathrm{CD} 20^{+}, \mathrm{CD} 22^{+}, \mathrm{CD} 10^{+}, \mathrm{CD} 23^{+}$indicative for an aggressive B-cell lymphoma. Based on this preliminary diagnosis, prephase treatment was escalated to $\mathrm{R}-\mathrm{CHOP}$ due to the extent of disease and to treat the EBV reactivation (rituximab $375 \mathrm{mg} / \mathrm{m}^{2}$ administered twice at an interval of one week, cyclophosphamide $150 \mathrm{mg} / \mathrm{m}^{2}$ in order to reach a complete dose of $750 \mathrm{mg} / \mathrm{m}^{2}$, doxorubicin $50 \mathrm{mg} / \mathrm{m}^{2}$ and vincristine $1.4 \mathrm{mg} / \mathrm{m}^{2}$ ).

Therapy was well tolerated. However, a follow-up TTE three days after administration revealed a sudden drop in LVEF to $35-40 \%$ with LV dilatation, akinesia of the apical and medial septal zones and hypokinesia of the medial segments. The right atrial mass was still present, and the RV showed normal contractility. The patient reported dyspnea on exertion, but no chest pain. Troponin $\mathrm{T}$ concentration was mildly elevated at $34 \mathrm{ng} / \mathrm{l}$ (reference $<14 \mathrm{ng} / \mathrm{l}$ ). Invasive cardiac catheterization was not performed in light of the atypical constellation of findings for acute myocardial infarction and a high likelihood of a lymphoma and/or lymphoma therapy associated cause of the symptoms. Nevertheless, a coronary computed tomography angiography 
was performed, which showed no evidence for coronary artery stenosis. Subsequently, therapy with a ß-blocker and an angiotensin-converting enzyme (ACE) inhibitor was initiated.

In the following days, immunohistochemical examination and fluorescence in situ hybridization (FISH) of the biopsy material from the submandibular lymph node/paranasal sinuses led to the diagnosis of BL with cells displaying a proliferation rate of virtually $100 \%$, an immunophenotype of $\mathrm{CD} 20^{+}, \mathrm{CD} 10^{+}, \mathrm{BCL}^{+}, \mathrm{MYC}^{+}$, $\mathrm{TdT}^{-}, \mathrm{BCL2}$, a strong positivity for a EBV and a rearrangement of $c-M Y C$ (Chr 8q24) on FISH analysis. There was no BL manifestation in the bone marrow. On day 10 following start of therapy, the patient was discharged with the diagnosis of a BL stage IV disease in a clinically much improved state (ECOG 1 ).

Chemotherapy was then continued analogous to block A1 of the German Multicenter Study Group on Adult Acute Lymphoblastic Leukemia (GMALL) B-ALL/NHL protocol as recommended by the local tumor board [10]. Interestingly, prior to treatment start the LVEF had normalized to $60 \%$ and the right atrial mass was no longer detectable on TTE, suggesting that the transient cardiac deterioration was indeed most likely attributable to the BL manifestation, which had regressed after R-CHOP therapy. We therefore decided to continue with the full dose of the A1 therapy block (rituximab $375 \mathrm{mg} / \mathrm{m}^{2}$, vincristine $2 \mathrm{mg}$, cytarabine $150 \mathrm{mg} / \mathrm{m}^{2}$ twice daily for two days, etoposide $100 \mathrm{mg} /$ $\mathrm{m}^{2}$ for two days, methotrexate (MTX) $1500 \mathrm{mg} / \mathrm{m}^{2}$, ifosfamide $800 \mathrm{mg} / \mathrm{m}^{2}$ for five days and dexamethasone $10 \mathrm{mg} / \mathrm{m}^{2}$ for five days; intrathecal prophylaxis with cytarabine $40 \mathrm{mg}$, dexamethasone $4 \mathrm{mg}$ and MTX $15 \mathrm{mg}$ was administered twice) while continuing to closely monitor cardiac function. After the second chemotherapy block, TTE again revealed a slight decrease in LVEF (45-50\%) with reduced longitudinal strain.

A follow-up TTE during the third cycle of chemotherapy showed a normal LVEF with borderline longitudinal strain and normal RV function. Notably, the thickening of the RV wall had vanished in comparison to the baseline exam.

Overall, the patient was treated with accumulating doses of chemotherapy according to protocol in a total of 7 cycles, and the LVEF remained normal after the initial recovery from acute dysfunction. CT staging revealed a complete remission after completion of chemotherapy. Subsequently, the patient underwent consolidating radiation of the mediastinum at a cumulative dose of $36 \mathrm{~Gy}$ in 13 fractions.

With a follow-up of 16 months after diagnosis, the patient is in continuous complete remission as of now.

\section{Discussion and conclusions}

$\mathrm{BL}$ is one of the most aggressive B-cell lymphomas with a rapid doubling time [1]. The case presented here is very unusual regarding its EBV-reactivation in a sporadic $\mathrm{BL}$ and its clinical presentation with extensive cardiac involvement.

EBV reactivation, as seen in our patient, is rarely seen in sporadic $\mathrm{BL}$ and much more frequent in the immunodeficiency-associated form [11]. However, negative testing for HIV ruled out acquired immune deficiency syndrome (AIDS) as a reason for immunosuppression and CD21-negativity favors the diagnosis of a sporadic BL form.

In 2016, Chan et al. published a review of all 22 cases of patients presenting with intracardiac BL manifestations reported in the English literature, and we found six additional cases that have been published since then. Patients were predominantly male and presented with similar symptoms as our patient, including fatigue, tachycardia and dyspnea. Information on administered chemotherapy is available for 18 cases. While about $2 / 3$ of patients receiving treatment responded to therapy, the rest died within days to weeks after diagnosis, with heart failure being a major contributing factor to the causes of death. On the other hand, all patients reported not to have received therapy died of the disease within days $[5,12-$ 17]. Thus, based on the very limited data available, rapid initiation of treatment-at the risk of severe acute cardiac insufficiency-seems to be the only potentially lifesaving option for affected patients. It remains uncertain whether an increased susceptibility to therapy-induced myocardial toxicity or intracardiac tumor cell lysis during treatment is responsible for cardiac failure.

Acrolein, a metabolite of cyclophosphamide, is known to have a direct toxic effect on endothelial cells and the myocardium [18]. Anthracyclines can also induce acute and late cardiotoxicity due to increased sympathetic stimulation, causing microvascular dysfunction as well as alterations in cytokine, free radical and catecholamine receptor levels. Acute toxicity usually manifests as disturbance in electrical conduction, while chronic toxicity can lead to reductions in LVEF [19-22]. While there is no universally-accepted clinical definition of chemotherapyassociated cardiomyopathy, general consensus entails a reduction of global LVEF $>10-15 \%$ [23].

The localized left ventricular akinesia observed in our patient shortly after initial R-CHOP therapy is therefore not typical for acute chemotherapy-induced toxicity. Furthermore, growing evidence suggests that low troponin levels after anthracycline therapy-as observed in our patient-are related to better cardiac outcomes due to limited myocardial injury [24]. We therefore believe that rapid intracardiac tumor lysis, rather than myocyte 
loss, mainly contributed to the decrease in LVEF in our patient, perhaps through to changes in the cardiac microcirculation and in energy metabolism. The sudden and successively smaller drops in LVEF observed periodically after the following chemotherapy cycles appear related to the amount of residual intracardiac lymphoma mass, culminating in a complete recovery of LVEF.

Interestingly, imaging at diagnosis showed predominant BL manifestations in the right heart, while the observed localized cardiac malfunction manifested in the left ventricle. We believe that BL infiltration too small to be picked up on $\mathrm{CT}$ or echocardiography was also present in the left ventricle, explaining these discrepancies. However, to elucidate the underlying mechanisms of acute cardiac insufficiency in patients with cardiac tumor involvement, preclinical studies are needed to explore possibilities beyond therapy-associated toxicity.

In conclusion, in the rare case of cardiovascular involvement secondary to $\mathrm{BL}$, rapid lymphoma control is crucial to prevent life-threatening hemodynamic instability. This case illustrates that a prompt treatment start can save affected patients' lives with full cardiovascular recovery despite transient deterioration of cardiac function.

\begin{abstract}
Abbreviations
ACE: Angiotensin-converting enzyme; B-ALL: B-lymphocytic acute lymphoid leukemia; BCL2: B-cell lymphoma 2; BCL6: B-cell lymphoma 6; BL: Burkitt Lymphoma; $\mathrm{CD}$ : Class of differentiation; $\mathrm{CT}$ : Computed tomography; EBV: Epstein Barr Virus; ECOG: Eastern Cooperative Oncology Group; FISH: Fluorescence in-situ hybridization; GMALL: The German Multicenter Study Group on Adult Acute Lymphoblastic Leukemia; HIV: Human immunodeficiency virus; LDH: Lactate dehydrogenase; LVEF: Left ventricular ejection fraction; MTX: Methotrexate; NHL: Non-Hodgkin's Lymphoma; R-CHOP: Chemotherapy regimen containing Rituximab, Cyclophosphamide, Doxorubicin, Vincristine and Prednisone; RVOT: Right ventricular outflow tract; TTE: Transthoracic echocardiogram.
\end{abstract}

\section{Acknowledgements}

We gratefully acknowledge the help of the Labor Berlin providing images of cytology. Additional affiliations of IN are Berlin Institute of Health at CharitéUniversitätsmedizin Berlin, BIH Center for Regenerative Therapies (BCRT), Berlin, Germany; Experimental and Clinical Research Center (ECRC), Berlin, Germany; and German Cancer Consortium (DKTK), Berlin, Germany.

\section{Authors' contributions}

MS drafted the manuscript and analyzed and interpreted the patient's data. ET edited the manuscript and analyzed and interpreted the patient's data. FB performed analyses of transthoracic echocardiograms and provided cardiological expertise. DG performed radiological analyses. AL, FD, LB and IN reviewed the clinical notes and edited the document. All authors have read and approved the final version of the manuscript.

\section{Funding}

Open Access funding enabled and organized by Projekt DEAL. MS and ET are participants in the BIH-Charité Junior Scientist Program funded by the CharitéUniversitätsmedizin Berlin and the Berlin Institute of Health. The program guarantees research-active physicians a competence-based residency training with a contractually regulated share of working time ("protected time") for clinical and translational research.

\section{Availability of data and materials}

The data analyzed are available from the corresponding author on reasonable request.

\section{Declarations}

Ethics approval and consent to participate

Not applicable.

\section{Consent for publication}

Written informed consent for publication of their clinical details and clinical images was obtained from the patient. A copy of the consent form is available for review by the Editor of this journal.

\section{Competing interests}

The authors declare that they have no competing interests.

\section{Author details}

'Department of Hematology, Oncology, and Tumor Immunology, CharitéUniversitätsmedizin Berlin, Corporate Member of Freie Universität Berlin and Humboldt-Universität zu Berlin, Berlin, Germany. ${ }^{2}$ Department of Cardiology, Charité-Universitätsmedizin Berlin, Corporate Member of Freie Universität Berlin and Humboldt-Universität zu Berlin, Berlin, Germany. ${ }^{3}$ Department of Radiology, Charité-Universitätsmedizin Berlin, Corporate Member of Freie Universität Berlin and Humboldt-Universität zu Berlin, Berlin, Germany.

Received: 25 November 2021 Accepted: 27 January 2022

Published online: 04 February 2022

\section{References}

1. Morton LM, Wang SS, Devesa SS, Hartge P, Weisenburger DD, Linet MS. Lymphoma incidence patterns by WHO subtype in the United States, 1992-2001. Blood. 2006;107(1):265-76.

2. Brady G, MacArthur GJ, Farrell PJ. Epstein-Barr virus and Burkitt lymphoma. J Clin Pathol. 2007;60(12):1397-402.

3. Mbulaiteye SM, Biggar RJ, Bhatia K, Linet MS, Devesa SS. Sporadic childhood Burkitt lymphoma incidence in the United States during 1992-2005. Pediatr Blood Cancer. 2009:53(3):366-70.

4. Gordon MJ, Danilova O, Spurgeon S, Danilov AV. Cardiac non-Hodgkin's lymphoma: clinical characteristics and trends in survival. Eur J Haematol. 2016;97(5):445-52.

5. Chan O, Igwe M, Breburda CS, Amar S. Burkitt lymphoma presenting as an intracardiac mass: case report and review of literature. Am J Case Rep. 2016:17:553-8.

6. Roberts WC, Glancy DL, DeVita VT Jr. Heart in malignant lymphoma (Hodgkin's disease, lymphosarcoma, reticulum cell sarcoma and mycosis fungoides). A study of 196 autopsy cases. Am J Cardiol. 1968;22(1):85-107.

7. McDonnell PJ, Mann RB, Bulkley BH. Involvement of the heart by malignant lymphoma: a clinicopathologic study. Cancer. 1982;49(5):944-51.

8. O'Mahony D, Peikarz RL, Bandettini WP, Arai AE, Wilson WH, Bates SE. Cardiac involvement with lymphoma: a review of the literature. Clin Lymphoma Myeloma. 2008;8(4):249-52

9. Oken MM, Creech RH, Tormey DC, Horton J, Davis TE, McFadden ET, et al. Toxicity and response criteria of the Eastern Cooperative Oncology Group. Am J Clin Oncol. 1982;5(6):649-55.

10. Hoelzer D, Walewski J, Döhner H, Viardot A, Hiddemann W, Spiekermann $\mathrm{K}$, et al. Improved outcome of adult Burkitt lymphoma/leukemia with rituximab and chemotherapy: report of a large prospective multicenter trial. Blood. 2014;124(26):3870-9.

11. Blum KA, Lozanski G, Byrd JC. Adult Burkitt leukemia and lymphoma. Blood. 2004;104(10):3009-20.

12. Koca Yozgat A, Ozyoruk D, Kacar D, Cetin II, Yaman Bajin I, Inan K, et al. Burkitt lymphoma presenting with intracardiac mass and tumor thrombosis in the anterior mediastinum with literature review. J Pediatr Hematol Oncol. 2019;41(3):e197-200. 
13. Vilaça A, Monteiro M, Pimentel T, Marques H. Intracardiac mass from Burkitt's lymphoma in an immunocompromised patient: a very rare form of presentation. BMJ Case Rep. 2017;2017.

14. Cadavid L, Sastoque JM, Gutiérrez C, Yabur M, Molina G. Primary osseous Burkitt lymphoma with nodal and intracardiac metastases in a child. Radiol Case Rep. 2017;12(1):185-90.

15. Vervloet DM, De Pauw M, Demulier L, Vercammen J, Terryn W, Steel E, et al. Aggressive extensive cardiac mass in an HIV-1-infected patient: should we go for comfort therapy? Acta Clin Belg. 2017;72(3):198-200

16. Tzachanis D, Dewar R, Luptakova K, Chang JD, Joyce RM. Primary cardiac burkitt lymphoma presenting with abdominal pain. Case Rep Hematol. 2014;2014:687598.

17. Kaul $P$, Javangula K. Burkitt lymphoma masquerading as cardiac tamponade. J Cardiothorac Surg. 2007;2:30.

18. Iqubal A, Iqubal MK, Sharma S, Ansari MA, Najmi AK, Ali SM, et al. Molecular mechanism involved in cyclophosphamide-induced cardiotoxicity: old drug with a new vision. Life Sci. 2019;218:112-31.

19. Pai VB, Nahata MC. Cardiotoxicity of chemotherapeutic agents: incidence, treatment and prevention. Drug Saf. 2000;22(4):263-302.

20. Jackson JA, Reeves JP, Muntz KH, Kruk D, Prough RA, Willerson JT, et al. Evaluation of free radical effects and catecholamine alterations in adriamycin cardiotoxicity. Am J Pathol. 1984;117(1):140-53.

21. Abdul Hamied TA, Parker D, Turk JL. Effects of adriamycin, 4-hydroperoxycyclophosphamide and ASTA Z 7557 (INN mafosfamide) on the release of IL-2 and IL-1 in vitro. Int J Immunopharmacol. 1987;9(3):355-61.

22. Ehrke MJ, Maccubbin D, Ryoyama K, Cohen SA, Mihich E. Correlation between adriamycin-induced augmentation of interleukin 2 production and of cell-mediated cytotoxicity in mice. Cancer Res. 1986;46(1):54-60.

23. Levis BE, Binkley PF, Shapiro CL. Cardiotoxic effects of anthracycline-based therapy: what is the evidence and what are the potential harms? Lancet Oncol. 2017:18(8):e445-56.

24. Simões R, Silva LM, Cruz ALVM, Fraga VG, de Paula SA, Gomes KB. Troponin as a cardiotoxicity marker in breast cancer patients receiving anthracycline-based chemotherapy: a narrative review. Biomed Pharmacother. 2018;107:989-96.

\section{Publisher's Note}

Springer Nature remains neutral with regard to jurisdictional claims in published maps and institutional affiliations.

Ready to submit your research? Choose BMC and benefit from:

- fast, convenient online submission

- thorough peer review by experienced researchers in your field

- rapid publication on acceptance

- support for research data, including large and complex data types

- gold Open Access which fosters wider collaboration and increased citations

- maximum visibility for your research: over $100 \mathrm{M}$ website views per year

At BMC, research is always in progress.

Learn more biomedcentral.com/submissions 